\title{
Discrepancy between perceived pain and cortical processing: A voxel- based morphometry and contact heat evoked potential study
}

\author{
Kramer JLK ${ }^{1-3}$, Jutzeler $\mathrm{CR}^{1}$, Haefeli J ${ }^{1,4}$, Curt $\mathrm{A}^{1}$, Freund $\mathrm{P}^{1,5-6}$
}

1. Spinal Cord Injury Center, University Hospital Balgrist, University of Zurich, Zurich, Switzerland

2. The Hulse Spinal Cord Injury Laboratory, Shepherd Center, Atlanta, Georgia, USA

3. ICORD/School of Kinesiology, University of British Columbia, Vancouver, British Columbia, Canada

4. Brain and Spinal Injury Center, Department of Neurological Surgery, University of California, San Francisco, USA

5. Wellcome Trust Centre for Neuroimaging, UCL Institute of Neurology, University College London, UK

6. Department of Brain Repair \& Rehabilitation, UCL Institute of Neurology, University College London, UK

\section{Corresponding author:}

John LK Kramer

ICORD

Blusson Spinal Cord Centre

818 W $10^{\text {th }}$ Ave

Vancouver, British Columbia, Canada

Tel.: +1.604.675.8876

E-mail: kramer@icord.org 


\section{Abstract}

Objectives: The purpose of this study was to determine if local gray and white matter volume variations between subjects could account for variability in responses to CHEP stimulation.

Methods: Structural magnetic resonance imaging was used to perform voxel-based morphometry (VBM) of gray and white matter in 30 neurologically healthy subjects. Contact heat stimulation was performed on the dorsum of the right hand at the base of the thumb. Evoked potentials were acquired from a vertex-recording electrode referenced to linked ears. Results: Controlling for age, total intracranial volume, and skull/scalp thickness, CHEP amplitude and pain rating were not significantly correlated between subjects. A VBM region of interest approach demonstrated a significant interaction between pain rating and N2 amplitude in the right insular cortex ( $p<0.05$, family-wise error corrected, FWE). In white matter, a significant interaction was localized in the right inferior frontal occipital fasciculus (IFOF, $p<0.05$ FWE). Conclusions: Accounting for gray matter volume in the right insular cortex, resulted in a significant relationship between CHEP amplitude and pain rating. Significance: This finding suggests that the discrepancy between pain ratings and the amplitude of evoked potentials is not solely related to measurement artifact, but rather attributable, in part, to anatomical differences between subjects.

Keywords: Contact heat evoked potentials; laser evoked potentials; insular; inferior frontal occipital fasciculus; gray matter; white matter. 


\subsection{Introduction}

At present, self-report (i.e., description and intensity) represents a clinical standard for the evaluation of pain. To detect subtle changes in somatosensory function, an objective assessment of pain is desirable. Electrophysiological analogues of conventional somatosensory evoked potentials acquired in response to electrical stimulation and central conduction in the dorsal columns, laser and contact heat evoked potentials (LEPs and CHEPs) represent objective methods to assess the integrity of small diameter afferents conveying temperature and pain sensation to the brain in the spinothalamic tract (Baumgartner et al., 2012). However, ratings as well as the amplitude of prominent cortical waveforms (e.g., N2P2) typically demonstrate high between-subject variability (Chen et al., 2006; Cruccu et al., 2008). Furthermore, while significant positive relationships between amplitude of evoked potentials and pain ratings have been reported (Beydoun et al., 1993; Chen et al., 2006; Garcia-Larrea et al., 1997; lannetti et al., 2005), this too is highly variable - some individuals rating low intensity to stimulation but demonstrating large amplitude cortical potentials, and vice versa. The dissociation between pain rating and amplitude of prominent cortical potentials is also evident within an individual, shown in response to repeated and predictable stimulation (lannetti et al., 2008). On one hand, these observations may be related, in part, to inherent difficulties rating noxious stimuli. For example, two individuals subjected to the same stimulation may lead to comparable EP amplitudes, but due to differences in their interpretation of scales used to rate pain intensity (e.g., 0-10), one individual reports an appreciably higher rating than the other. In such a case, the mismatch between CHEP/LEP amplitude and rating is prominently a function of measurement artifact, unrelated to differences in anatomy and physiology. However, central pain patients with thermal hyperalgesia demonstrate high prevalence of a similar phenomenon, often reporting high ratings coupled with small amplitude evoked potentials (Casey et al., 1996; Garcia-Larrea et al., 2002; Tinazzi et al., 2009; Wu et al., 1999). Collectively, these observations in healthy subjects and central pain patients raise the question whether a neural substrate underlies the discrepancy between ratings and evoked potential amplitudes. 
By accounting for differences in sensitivity to noxious thermal stimuli (Emerson et al., 2014; Erpelding et al., 2012; Grant et al., 2010; Tseng et al., 2013), ability to modulate pain (Piche et al., 2013; Stankewitz et al., 2013; Teutsch et al., 2008), as well as the amplitude of evoked cortical responses to non-noxious afferent stimuli (Fjell et al., 2007; Liem et al., 2012; Muthukumaraswamy et al., 2010), structural magnetic resonance imaging (MRI) has highlighted an important relationship between normal brain anatomy and sensory function. Based on this knowledge, we intended to address the question whether between-subject variability in cortical structure could account for differences in responses to contact heat stimulation in healthy subjects. Specifically, we were interested in determining if estimates of gray and white matter volume explained, in part, why an individual perceived stimulation as low/high but generated an evoked cortical potential that was large/small. To this end, voxel-based morphometry (VBM) was used to explore associations between pain rating and CHEP amplitude in gray and white matter.

\subsection{Methods}

\subsection{Subjects}

Thirty neurologically healthy subjects participated in this study (13 females, 17 males). All subjects were prescreened for MRI contraindications and reported no acute or chronic pain at the time of examination. Subjects provided written informed consent and all procedures described below were in accordance with the Declaration of Helsinki, and approved by research ethics board at the University of Zurich (ref. number: EK-04/2006).

\subsection{Study Protocol}

\subsubsection{Acquisition of pain rating and contact heat evoked potentials (CHEPS)}

CHEPs and pain rating to contact heat stimuli were recorded following stimulation of the dorsal surface of the $\mathrm{C} 6$ dermatome at the base of the right thumb using the Pathway Pain and Sensory Evaluation System (Medoc Advanced Medical Systems ${ }^{\circledR}$ ). 10 contact heat stimuli were delivered from a baseline temperature of $35^{\circ} \mathrm{C}$ to a peak temperature of $52^{\circ} \mathrm{C}$, at an inter-pulse 
interval of 8-12 seconds. To familiarize subjects and limit the startle effect, individuals were exposed to contact heat stimuli on an untested site (e.g., forearm) before acquisition of evoked potentials. The contact heat stimulation thermode was repositioned (i.e. variable stimulation protocol) after each stimulus to reduce receptor fatigue. In response to an audio cue presented 2 seconds after contact heat stimulation, subjects rated perceived intensity according to a 0-10 numerical rating scale (NRS). Subjects were instructed not to blink in response to the stimulation, and withhold from blinking until hearing the audio cue. N2P2 was acquired from an active vertex-recording electrode $(\mathrm{Cz})$ referenced to both earlobes (A1-A2). Previous studies have adopted a similar electrode configuration (i.e., single recording channel) for the acquisition of N2P2 (Chao et al., 2008; Chao et al., 2007; Chen et al., 2006), reporting significant intra-subject (i.e., test-retest) reliability for N2P2 amplitude (Kramer et al., 2012; Ruscheweyh et al., 2013). A ground strap electrode was secured on the upper arm of the stimulating side. A pre-trigger period of $100 \mathrm{~ms}$ preceded each recording, followed by a $1500 \mathrm{~ms}$ post-trigger, for a total $2000 \mathrm{~ms}$ epoch. All signals were sampled at $2,000 \mathrm{~Hz}$ and amplified $(20,000 \mathrm{x})$. Each stimulus was manually reviewed for artifiact.

\subsubsection{Automated detection of N2P2 amplitude}

To enhance the signal-to-noise ratio of CHEPs, vertex recordings were bandpass (1$30 \mathrm{~Hz}$ ) and wavelet filtered for automated detection of N2 and P2 amplitudes. The aim of wavelet filtering is to improve the signal to noise ratio, so as to allow automated detection of peak waveforms. Based on wavelet filtered CHEPs, single trial averaged N2P2, N2, and P2 amplitudes were determined. The advantage of single trial averaging compared to across trial averaging is that latency jitter does not affect the amplitude of responses. Rather, a measure of amplitude is extracted for each stimulus, and averaged across the total number of stimulations. In order to perform an unbiased single trial analysis of N2P2 amplitude from wavelet filtered CHEPs, an automated approach utilizing multiple linear regression with a dispersion term was performed in MatLab (Mathworks) (Hu et al., 2011; Hu et al., 2010). We applied the same techniques as described previously for CHEPs (Kramer et al., 2013).

\subsubsection{Magnetic resonance image sequence}


Using a Philips 3T Ingenia, a 3D-GRE T1-weighted (T1w) sequence was used to acquire a whole-brain, structural scan optimized for simultaneous assessment of the brain and spinal cord (Freund et al., 2010). The imaging parameters were: isotropic $1 \mathrm{~mm}^{3}$ resolution, field of view $256 \times 256 \mathrm{~mm}^{2}$, matrix $256 \times 256,180$ sagittal partitions, repetition time $=7.15 \mathrm{~ms}$, echo time $=3.29 \mathrm{~ms}$, inversion time $=858.65 \mathrm{~ms}$, flip angle $8^{\circ}$, fat saturation, bandwidth $250 \mathrm{~Hz} /$ pixel and a scan time of $6 \mathrm{~min} 31 \mathrm{~s}$. Prior to VBM analysis, MRI data from each subject was visually screened for movement artifacts.

\subsubsection{Voxel-based morphometry (VBM)}

To assess voxel-wise associations of gray and white matter volumes with N2, P2, and N2P2 amplitudes and pain ratings, VBM was performed in the framework of Statistical Parametric Mapping 8 (SPM8, http://www.fil.ion.ucl.ac.uk/spm/software/spm8/), applied on the acquired T1w 3D volumetric MRI data (Ashburner and Friston, 2000). First, a unified model inversion (unified segmentation) was used for bias correction and segmentation of MRI data into gray and white matter, and cerebrospinal fluid. Then Dartel was used to warp the gray and white matter segments into an optimal (average) space (Ashburner, 2007). The resulting gray and white matter images were modulated and affine transformed to Montreal Neurological Institute (MNI) space and smoothed using an isotropic Gaussian kernel with $6 \mathrm{~mm}$ full width at half-maximum. After each post-processing step, the alignment of images (i.e., gray and white matter) was verified to ensure the accuracy of fully automated post-processing in SPM.

\subsection{Analysis and Statistics}

To determine the relationship between CHEP parameters and rating, partial correlations were examined. This analysis was performed in IBM SPSS Statistics (V.19.0), and included controlling for age, skull/scalp thickness, as well as total intracranial volume (TIV). Two-tailed significance was determined at $p<0.05$.

General linear models in SPM were utilized to assess associations between the covariates of interest (i.e., automated single trial averaged N2, P2, and N2P2 amplitudes and corresponding pain rating to contact heat stimulation) and local grey and white matter 
volumes. This is a routine procedure within the SPM framework, and operates irrespectively of whether data is derived from functional or anatomical imaging. To account for possible nonspecific effects on regional gray and white matter volumes, TIV, skull/scalp thickness, and age were included as nuisance variables. Skull/scalp thickness was estimated at the vertex from anatomical T1w scans. Using Gaussian random field theory, F-contrasts were examined to address the interaction between CHEP amplitude and pain rating in gray and white matter. In each model, a CHEP amplitude parameter (N2P2, N2, or P2), pain rating, and all nuisance variables (i.e., age, TIV, skull/scalp thickness) were included. Initially, a whole brain analysis of gray and white matter was performed. For a more directed approach of gray matter, regions of interest (ROIs) were then examined. The ROIs included the primary and secondary somatosensory cortices, anterior and mid cingulate gyrus, and insular cortex - that is, areas previously shown to be associated with noxious contact heat stimulation (Haefeli et al., 2014; Haefeli et al., 2013; Tseng et al., 2010). ROIs and the white matter mask were created using the Pick Atlas in SPM8 (Maldjian et al., 2004; Maldjian et al., 2003). The John Hopkins University (JHU) White-Matter Tractography Atlas was used to identify the location of significant peak voxels in the white matter (Hua et al., 2008). Associated p-values were corrected for multiple comparisons across the whole brain, and in each individual ROI (Family Wise-Error, FWE). Significance was set at $\mathrm{p}<0.05$ (FWE-corrected).

To determine if estimates of gray or white matter volume improved the relationship between CHEP amplitude and pain rating, partial correlations were again examined in SPSS. In addition to age, TIV, and skull/scalp thickness, gray and white matter contrast estimates extracted from significant peak voxels were included in this analysis.

\subsection{Results}

\subsection{Correlations between CHEP amplitude and pain rating: Absolute and relative relationship}

A representative example of CHEPs (single trial and grand average for one subject) is shown in Figure 1. Partial correlations of CHEP amplitude parameters and pain rating, controlling for age, TIV, and skull/scalp thickness, are shown in Table 1. While positive, all correlations between CHEP amplitude (N2, P2 or N2P2) and rating were insignificant ( $>>0.05)$. 
Further highlighting the poor correlation between CHEP amplitude and pain rating, among subjects demonstrating higher than average amplitudes (+1SD, $n=5)$, only 1 subject also reported a similar trend in pain rating (i.e., NRS was +1SD greater than the average). Of those individuals with CHEP amplitudes less than average $(-1 S D, n=6)$, only 2 also reported ratings below average. In total, only $50 \%$ of subjects demonstrated the same relative relationship between CHEP amplitude and pain rating (i.e., low/average/high rating, small/average/large amplitudes, see Table 2).

\subsection{Voxel-based morphometry}

At the whole brain level analysis in gray matter, no significant associations for N2P2, N2, or P2 amplitude with pain rating were identified. However, ipsilateral to contact heat stimulation, a significant interaction between $\mathrm{N} 2$ amplitude and pain rating $(\mathrm{p}=0.034, \mathrm{~F}=14.35$, $\mathrm{z}=3.78, \mathrm{~K}_{\mathrm{e}}=18$; Figure $2 \mathrm{~A}$ ) was observed in the right insular cortex (MNI: $\left.x=48, y=7, \mathrm{z}=6\right)$. Put simply, increased gray matter volume in the insular cortex was associated with larger N2 amplitudes and lower pain ratings. In white matter, also ipsilateral to contact heat stimulation, there was a significant association between pain rating and N2P2 amplitude $(p=0.025, F=29.43$, $\mathrm{z}=4.96, \mathrm{~K}_{\mathrm{e}}=414$ ) in the inferior frontal-occipital fasciculus (IFOF; MNI: $\mathrm{x}=33, \mathrm{y}=-35, \mathrm{z}=9$; Figure 2B). The association was only significant for N2P2 amplitude (positive, such that larger amplitudes were associated with greater white matter volume).

Effect of controlling for gray/white matter volume on correlations between pain rating and CHEP amplitude

Accounting for age, skull/scalp thickness, TIV, and contrast estimates of gray matter volume in the right insular resulted in a significant relationship between N2 amplitude and rating (Figure 3). In contrast, controlling for age, skull/scalp thickness, TIV, and estimates of white matter volume in the IFOF did not improve the correlation between N2P2 amplitude and pain rating.

\subsection{Discussion}


In the present study, individuals with higher volumetric estimates of gray matter in the right insular cortex and white matter in the right IFOF were found to be associated with larger amplitude CHEPs (N2 and N2P2, respectively, Figure 2). Furthermore, gray matter estimates in the right insular cortex, ipsilateral to contact heat stimulation, improved between subject associations for CHEP amplitude and pain rating, such that higher volumes were associated with larger N2 amplitudes and lower pain ratings. Building on previous studies (Emerson et al., 2014; Erpelding et al., 2012; Grant et al., 2010), these findings collectively support that structural differences in the brain may contribute to variability in physiological responses to noxious stimulation.

\subsection{Role of the insular cortex in the generation of CHEPs and pain}

There is strong rationale for including the insular cortex in our analysis. Based on neuroimaging studies (e.g., fMRI), the operculoinsular cortex has been proposed as fundamentally involved in the encoding of the intensity of perceived pain (Peyron et al., 2000). To our knowledge, we are the first to demonstrate that structure in the insular cortex may account for the apparent discrepancy between the amplitude of sensory evoked potentials to noxious stimuli and rating of perceived intensity - that is, rating stimulation as high/low and CHEPs that are small/large. Wu and colleagues proposed an imbalance in medial (i.e., sensoryaffective) and lateral pain systems (i.e., sensory-discriminative) underlying the dissociation between perceived intensity and LEP amplitude (Wu et al., 1999). This explanation fits with our anatomical findings, as the insular cortex is a prominent structure in the medial pain system.

\subsection{Lateralized effects in the right hemisphere}

In agreement with gray matter findings in an a priori region of interest, significant associations between CHEP amplitude and white matter were lateralized to the right hemisphere, localized in the IFOF. The IFOF represents associative nerve fibers connecting occipital, temporal, parietal, and frontal cortical areas (Forkel et al., 2014; Sarubbo et al., 2013). Comparatively, the relationship in white matter appears much stronger than in gray matter significant on the whole-brain level (i.e., bilateral white matter hemispheres). However, associations were only evident with regards to CHEPs (i.e., N2P2), and did not improve the 
relationship between amplitude and rating (Figure 2). In general, anatomical variations in the right IFOF, as well as the right insular cortex may contribute to individual differences in CHEP amplitudes through a mechanism non-specific to nociception (e.g., attention and arousal) (Beydoun et al., 1993; Garcia-Larrea et al., 1997). Indeed, both are prominent white and gray matter structures in the "ventral attention network" (Eckert et al., 2009; Umarova et al., 2010; Yin et al., 2013) - a group of temporal-parietal and ventral frontal areas lateralized to the right side of the brain, which play pivotal roles in saliency detection. Although speculative, higher volumes in areas in the ventral attention network may provide individuals an advantage to perceive afferent stimuli as more salient, in turn generating larger amplitude CHEPs compared to individuals with smaller volumes. Several recent studies have highlighted the importance of stimulation saliency in the generation of LEPS - demonstrating a unique capacity to dissociate amplitudes and ratings when stimuli are delivered at short, fixed inter-pulse intervals (lannetti et al., 2008).

\subsection{Limitations}

Important to note in the current study are potential limitations. Based on a priori hypotheses, we employed a ROI approach to examine gray matter volume. From the many brain areas involved in the processing of noxious stimuli, areas were selected based on overlap with prominent structures active in response to noxious contact heat stimulation (Haefeli et al., 2014). Despite a relatively small sample size $(n=30)$, significant associations in a gray matter region (i.e., insular) were detected. While these effects are meaningful, we cannot rule out that other gray matter areas also underlie the dissociation between CHEPs and perceived intensity, and that we did not have sufficient power to detect these relationships.

An additional limitation of our study is that only the amplitude of the vertex N2P2 was considered in examining associations between CHEPs and gray/white matter volume, and only in response to noxious stimulation. At present, N2P2 represents the most clinically viable waveform to assess the integrity of spinothalamic tract conduction and cortical processing - the earlier, lateralized N1 component difficult to interpret following conventional contact heat stimulating techniques (i.e., low signal to noise ratio) (Kramer et al., 2013). While N1 may be 
less subject to cognitive factors, there is still considerable between subject variability, and the correlation with pain rating remains low (i.e., is a poor objective measure of pain). Associations with cortical anatomy may be more readily detected by examining N1 amplitudes, including in the primary somatosensory cortex (Valentini et al., 2012).

The present study also did not account for other factors that may potentially contribute to variability in responses to pain, including levels of attention and arousal (Arntz and de Jong, 1993; Eccleston and Crombez, 1999; Kenntner-Mabiala et al., 2008). Interestingly, reductions in arousal have been shown to lead to dissociation of behavioral and physiological responses, evidenced by reduced LEP amplitudes and higher pain ratings following sleep restriction (Tiede et al., 2010). However, it is also important to consider that, from a statistical perspective, a confounding variable must be associated with not only the explanatory variable of interest (e.g., pain), but also the outcome variable of interest (e.g., brain volume). While several factors that we did not control for may affect individual responses to noxious stimuli, if these are not also associated with brain volume, they will not materially affect statistical significance.

\subsection{Conclusion}

In conclusion, our findings demonstrate a significant association between pain rating and CHEP amplitude in local gray and white matter volume. These findings provide an anatomical substrate to explain the discrepancy between the amplitude of cortical evoked potentials in response to noxious stimulation, and pain rating. In a broader context, our findings provide further evidence that between-subject differences in brain anatomy can account for variations in response to sensory stimuli. 


\section{Acknowledgments}

We are thankful for the technical support of Dr. Roger Luechinger. John Kramer was supported by the International Foundation for Research in Paraplegia - Zurich and is a Michael Smith Foundation for Health Research and Rick Hansen Institute Scholar. The project was funded by the Swiss National Science Foundation. 


\section{References}

Arntz, A., and de Jong, P. (1993). Anxiety, attention and pain. J Psychosom Res 37, 423-431. Ashburner, J. (2007). A fast diffeomorphic image registration algorithm. Neuroimage 38, 95113.

Ashburner, J., and Friston, K.J. (2000). Voxel-based morphometry--the methods. Neuroimage $11,805-821$.

Baumgartner, U., Greffrath, W., and Treede, R.D. (2012). Contact heat and cold, mechanical, electrical and chemical stimuli to elicit small fiber-evoked potentials: merits and limitations for basic science and clinical use. Neurophysiol Clin 42, 267-280.

Beydoun, A., Morrow, T.J., Shen, J.F., and Casey, K.L. (1993). Variability of laser-evoked potentials: attention, arousal and lateralized differences. Electroencephalogr Clin Neurophysiol 88, 173-181.

Casey, K.L., Beydoun, A., Boivie, J., Sjolund, B., Holmgren, H., Leijon, G., Morrow, T.J., and Rosen, I. (1996). Laser-evoked cerebral potentials and sensory function in patients with central pain. Pain 64, 485-491.

Chao, C.C., Hsieh, S.C., Tseng, M.T., Chang, Y.C., and Hsieh, S.T. (2008). Patterns of contact heat evoked potentials (CHEP) in neuropathy with skin denervation: correlation of CHEP amplitude with intraepidermal nerve fiber density. Clin Neurophysiol 119, 653-661.

Chao, C.C., Hsieh, S.T., Chiu, M.J., Tseng, M.T., and Chang, Y.C. (2007). Effects of aging on contact heat-evoked potentials: the physiological assessment of thermal perception. Muscle Nerve 36, 30-38.

Chen, I.A., Hung, S.W., Chen, Y.H., Lim, S.N., Tsai, Y.T., Hsiao, C.L., Hsieh, H.Y., and Wu, T. (2006). Contact heat evoked potentials in normal subjects. Acta Neurol Taiwan 15, 184-191.

Cruccu, G., Aminoff, M.J., Curio, G., Guerit, J.M., Kakigi, R., Mauguiere, F., Rossini, P.M., Treede, R.D., and Garcia-Larrea, L. (2008). Recommendations for the clinical use of somatosensoryevoked potentials. Clin Neurophysiol 119, 1705-1719.

Eccleston, C., and Crombez, G. (1999). Pain demands attention: a cognitive-affective model of the interruptive function of pain. Psychol Bull 125, 356-366.

Eckert, M.A., Menon, V., Walczak, A., Ahlstrom, J., Denslow, S., Horwitz, A., and Dubno, J.R. (2009). At the heart of the ventral attention system: the right anterior insula. Hum Brain Mapp 30, 2530-2541.

Emerson, N.M., Zeidan, F., Lobanov, O.V., Hadsel, M.S., Martucci, K.T., Quevedo, A.S., Starr, C.J., Nahman-Averbuch, H., Weissman-Fogel, I., Granovsky, Y., et al. (2014). Pain sensitivity is inversely related to regional grey matter density in the brain. Pain 155, 566-573.

Erpelding, N., Moayedi, M., and Davis, K.D. (2012). Cortical thickness correlates of pain and temperature sensitivity. Pain 153, 1602-1609.

Fjell, A.M., Walhovd, K.B., Fischl, B., and Reinvang, I. (2007). Cognitive function, P3a/P3b brain potentials, and cortical thickness in aging. Hum Brain Mapp 28, 1098-1116.

Forkel, S.J., Thiebaut de Schotten, M., Kawadler, J.M., Dell'Acqua, F., Danek, A., and Catani, M. (2014). The anatomy of fronto-occipital connections from early blunt dissections to contemporary tractography. Cortex 56, 73-84. 
Freund, P.A., Dalton, C., Wheeler-Kingshott, C.A., Glensman, J., Bradbury, D., Thompson, A.J., and Weiskopf, N. (2010). Method for simultaneous voxel-based morphometry of the brain and cervical spinal cord area measurements using 3D-MDEFT. J Magn Reson Imaging 32, 1242-1247. Garcia-Larrea, L., Convers, P., Magnin, M., Andre-Obadia, N., Peyron, R., Laurent, B., and Mauguiere, F. (2002). Laser-evoked potential abnormalities in central pain patients: the influence of spontaneous and provoked pain. Brain 125, 2766-2781.

Garcia-Larrea, L., Peyron, R., Laurent, B., and Mauguiere, F. (1997). Association and dissociation between laser-evoked potentials and pain perception. Neuroreport 8, 3785-3789.

Grant, J.A., Courtemanche, J., Duerden, E.G., Duncan, G.H., and Rainville, P. (2010). Cortical thickness and pain sensitivity in zen meditators. Emotion 10, 43-53.

Haefeli, J., Freund, P., Kramer, J.L., Blum, J., Luechinger, R., and Curt, A. (2014). Differences in cortical coding of heat evoked pain beyond the perceived intensity: an fMRI and EEG study. Hum Brain Mapp 35, 1379-1389.

Haefeli, J.S., Blum, J., Steeves, J.D., Kramer, J.L., and Curt, A.E. (2013). Differences in spinothalamic function of cervical and thoracic dermatomes: insights using contact heat evoked potentials. J Clin Neurophysiol 30, 291-298.

Hu, L., Liang, M., Mouraux, A., Wise, R.G., Hu, Y., and lannetti, G.D. (2011). Taking into account latency, amplitude, and morphology: improved estimation of single-trial ERPs by wavelet filtering and multiple linear regression. J Neurophysiol 106, 3216-3229.

Hu, L., Mouraux, A., Hu, Y., and lannetti, G.D. (2010). A novel approach for enhancing the signalto-noise ratio and detecting automatically event-related potentials (ERPs) in single trials.

Neuroimage 50, 99-111.

Hua, K., Zhang, J., Wakana, S., Jiang, H., Li, X., Reich, D.S., Calabresi, P.A., Pekar, J.J., van Zijl, P.C., and Mori, S. (2008). Tract probability maps in stereotaxic spaces: analyses of white matter anatomy and tract-specific quantification. Neuroimage 39, 336-347.

lannetti, G.D., Hughes, N.P., Lee, M.C., and Mouraux, A. (2008). Determinants of laser-evoked EEG responses: pain perception or stimulus saliency? J Neurophysiol 100, 815-828.

lannetti, G.D., Zambreanu, L., Cruccu, G., and Tracey, I. (2005). Operculoinsular cortex encodes pain intensity at the earliest stages of cortical processing as indicated by amplitude of laserevoked potentials in humans. Neuroscience 131, 199-208.

Kenntner-Mabiala, R., Andreatta, M., Wieser, M.J., Muhlberger, A., and Pauli, P. (2008). Distinct effects of attention and affect on pain perception and somatosensory evoked potentials. Biol Psychol 78, 114-122.

Kramer, J.L., Haefeli, J., Jutzeler, C.R., Steeves, J.D., and Curt, A. (2013). Improving the acquisition of nociceptive evoked potentials without causing more pain. Pain 154, 235-241. Kramer, J.L., Taylor, P., Haefeli, J., Blum, J., Zariffa, J., Curt, A., and Steeves, J. (2012). Test-retest reliability of contact heat-evoked potentials from cervical dermatomes. J Clin Neurophysiol 29, 70-75.

Liem, F., Zaehle, T., Burkhard, A., Jancke, L., and Meyer, M. (2012). Cortical thickness of supratemporal plane predicts auditory N1 amplitude. Neuroreport 23, 1026-1030. Maldjian, J.A., Laurienti, P.J., and Burdette, J.H. (2004). Precentral gyrus discrepancy in electronic versions of the Talairach atlas. Neuroimage 21, 450-455. 
Maldjian, J.A., Laurienti, P.J., Kraft, R.A., and Burdette, J.H. (2003). An automated method for neuroanatomic and cytoarchitectonic atlas-based interrogation of $\mathrm{fMRI}$ data sets. Neuroimage 19, 1233-1239.

Muthukumaraswamy, S.D., Singh, K.D., Swettenham, J.B., and Jones, D.K. (2010). Visual gamma oscillations and evoked responses: variability, repeatability and structural MRI correlates.

Neuroimage 49, 3349-3357.

Peyron, R., Laurent, B., and Garcia-Larrea, L. (2000). Functional imaging of brain responses to pain. A review and meta-analysis (2000). Neurophysiol Clin 30, 263-288.

Piche, M., Chen, J.I., Roy, M., Poitras, P., Bouin, M., and Rainville, P. (2013). Thicker posterior insula is associated with disease duration in women with irritable bowel syndrome (IBS) whereas thicker orbitofrontal cortex predicts reduced pain inhibition in both IBS patients and controls. J Pain 14, 1217-1226.

Ruscheweyh, R., Emptmeyer, K., Putzer, D., Kropp, P., and Marziniak, M. (2013). Reproducibility of contact heat evoked potentials (CHEPs) over a 6 months interval. Clin Neurophysiol 124, 2242-2247.

Sarubbo, S., De Benedictis, A., Maldonado, I.L., Basso, G., and Duffau, H. (2013). Frontal terminations for the inferior fronto-occipital fascicle: anatomical dissection, DTI study and functional considerations on a multi-component bundle. Brain Struct Funct 218, 21-37. Stankewitz, A., Valet, M., Schulz, E., Woller, A., Sprenger, T., Vogel, D., Zimmer, C., Muhlau, M., and Tolle, T.R. (2013). Pain sensitisers exhibit grey matter changes after repetitive pain exposure: a longitudinal voxel-based morphometry study. Pain 154, 1732-1737.

Teutsch, S., Herken, W., Bingel, U., Schoell, E., and May, A. (2008). Changes in brain gray matter due to repetitive painful stimulation. Neuroimage 42, 845-849.

Tiede, W., Magerl, W., Baumgartner, U., Durrer, B., Ehlert, U., and Treede, R.D. (2010). Sleep restriction attenuates amplitudes and attentional modulation of pain-related evoked potentials, but augments pain ratings in healthy volunteers. Pain 148, 36-42.

Tinazzi, M., Recchia, S., Simonetto, S., Defazio, G., Tamburin, S., Moretto, G., Fiaschi, A., Miliucci, R., and Valeriani, M. (2009). Hyperalgesia and laser evoked potentials alterations in hemiparkinson: evidence for an abnormal nociceptive processing. J Neurol Sci 276, 153-158. Tseng, M.T., Chiang, M.C., Yazhuo, K., Chao, C.C., Tseng, W.Y., and Hsieh, S.T. (2013). Effect of aging on the cerebral processing of thermal pain in the human brain. Pain 154, 2120-2129.

Tseng, M.T., Tseng, W.Y., Chao, C.C., Lin, H.E., and Hsieh, S.T. (2010). Distinct and shared cerebral activations in processing innocuous versus noxious contact heat revealed by functional magnetic resonance imaging. Hum Brain Mapp 31, 743-757.

Umarova, R.M., Saur, D., Schnell, S., Kaller, C.P., Vry, M.S., Glauche, V., Rijntjes, M., Hennig, J., Kiselev, V., and Weiller, C. (2010). Structural connectivity for visuospatial attention: significance of ventral pathways. Cereb Cortex 20, 121-129.

Valentini, E., Hu, L., Chakrabarti, B., Hu, Y., Aglioti, S.M., and lannetti, G.D. (2012). The primary somatosensory cortex largely contributes to the early part of the cortical response elicited by nociceptive stimuli. Neuroimage 59, 1571-1581.

Wu, Q., Garcia-Larrea, L., Mertens, P., Beschet, A., Sindou, M., and Mauguiere, F. (1999). Hyperalgesia with reduced laser evoked potentials in neuropathic pain. Pain 80, 209-214. 
Yin, X., Han, Y., Ge, H., Xu, W., Huang, R., Zhang, D., Xu, J., Fan, L., Pang, Z., and Liu, S. (2013). Inferior frontal white matter asymmetry correlates with executive control of attention. Hum Brain Mapp 34, 796-813. 


\section{Figure Legends}

Fig.1: Representative CHEP for a healthy subject, before and after wavelet filtering for automated single trial averaging. Single trials are shown in addition to the average waveforms.

Fig. 2: Statistical parametric maps illustrating the association between pain rating and CHEP amplitude in gray matter (A) and white matter (B). For display purposes only, shown at $p<0.01$ (FWE-uncorrected). Note that while N2 and N2P2 amplitude demonstrates positive relationships in gray and white matter, only in gray matter does rating appear negatively related to volumetric estimates. IFOF, inferior frontal-occipital fasciculus.

Fig. 3: The relationship between N2 amplitude and pain rating corrected for age, TIV, and skull/scalp thickness (top panel), and gray matter volume in the insular cortex (bottom panel). Note that the relationship between $\mathrm{N} 2$ amplitude and rating is improved by inclusion of insular gray matter volume estimates. TIV, total intracranial volume. 


\section{Tables}

Table 1. Summary demographics and CHEP outcomes.

$\begin{array}{lcc} & \text { Mean } & \pm S D \\ \text { Age } & 33.00 & 11.57 \\ \text { Skull/scalp thickness }(\mathrm{mm}) & 7.79 & 0.80 \\ \text { Total intracranial volume }(\mathrm{L}) & 1.55 & 0.16 \\ \text { Pain rating } & 4.65 & 2.09 \\ \text { CHEP amplitude }(\mu \mathrm{V}) & & \\ \quad \mathrm{N} 2 \mathrm{P} 2 & 30.16 & 13.95 \\ \mathrm{~N} 2 & 15.99 & 8.04 \\ \mathrm{P} 2 & 14.17 & 6.90\end{array}$

Partial correlations ( $r$-values)

(controlled for age, skull/scalp thickness, and TIV)

$\begin{array}{ccc} & \text { Rating } & \text { p-value } \\ \text { N2P2 } & 0.265 & 0.158 \\ \text { N2 } & 0.243 & 0.196 \\ \text { P2 } & 0.241 & 0.199\end{array}$


Table 2. Relative CHEP amplitude (N2P2) and pain rating ( $n=30)$.

\begin{tabular}{|c|c|c|c|c|}
\hline & \multicolumn{3}{|c|}{ CHEPs $(n=)$} \\
\hline & & Average & Higher & Lower \\
\hline \multirow{3}{*}{ 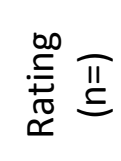 } & Average & 12 & 3 & 3 \\
\hline & Higher & 3 & 1 & 1 \\
\hline & Lower & 4 & 1 & 2 \\
\hline
\end{tabular}

No difference, +/- 1SD

Higher, $>1 S D$

Lower, <1SD

:relative CHEPs and pain rating are in agreement 


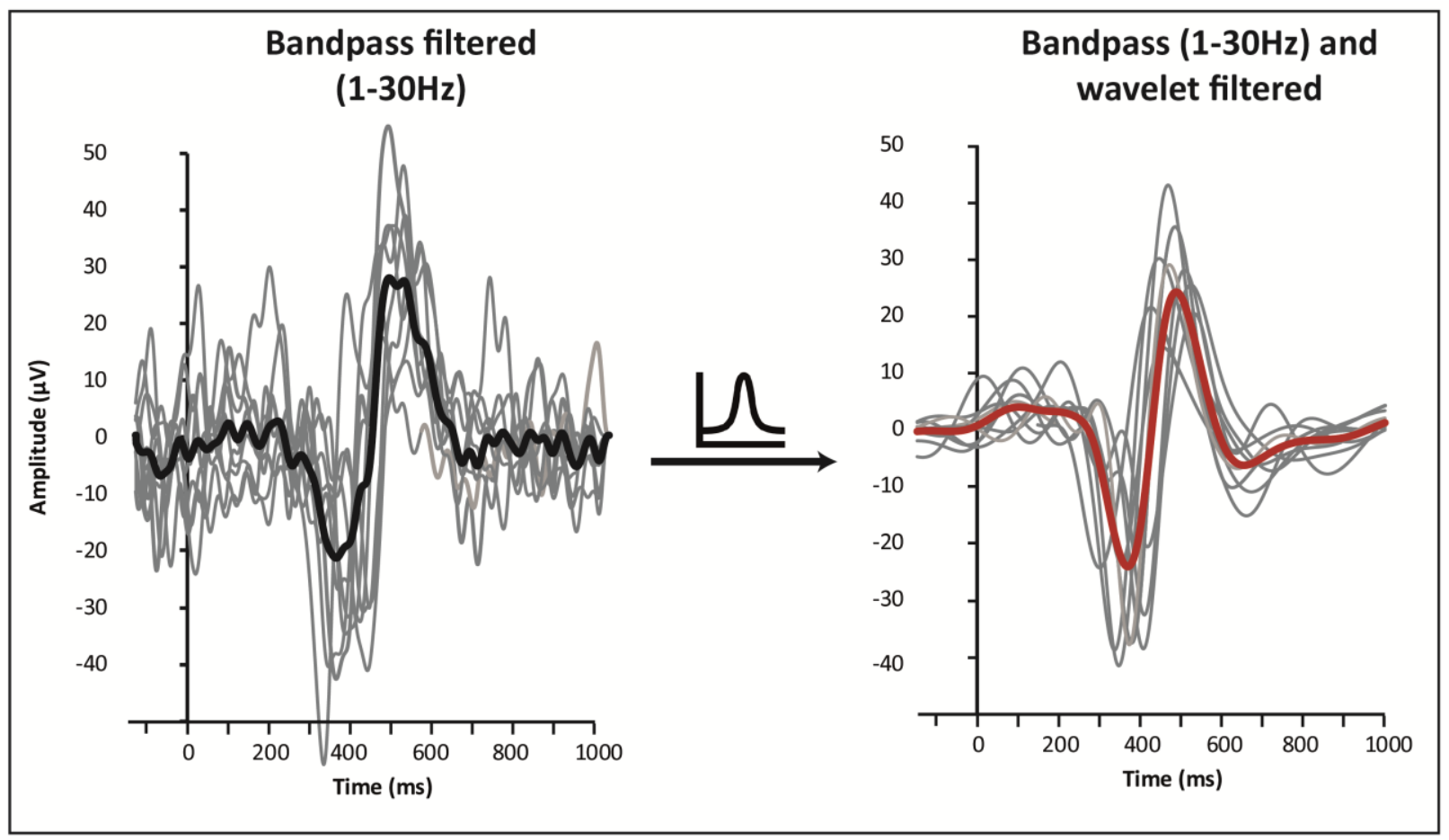



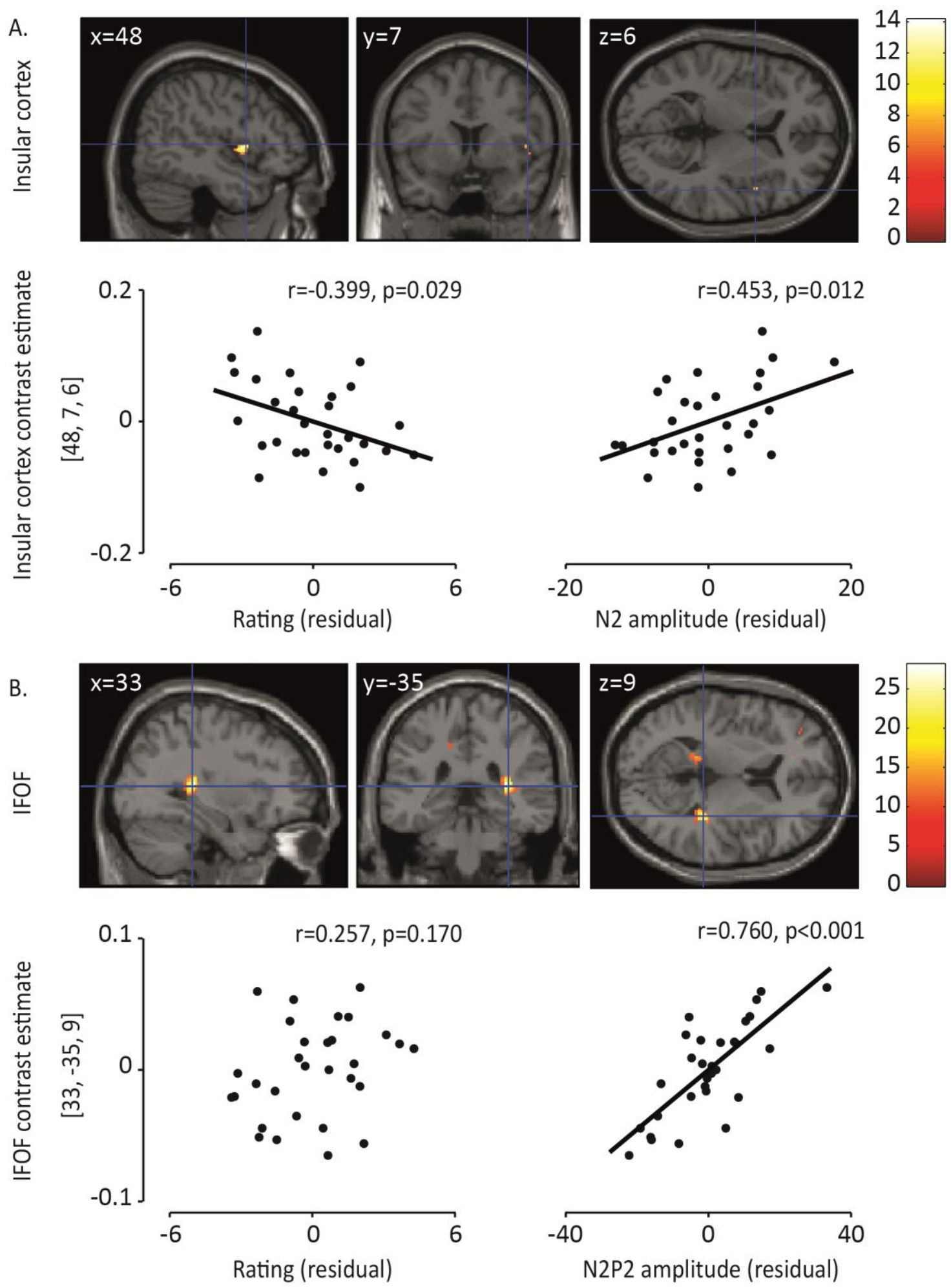


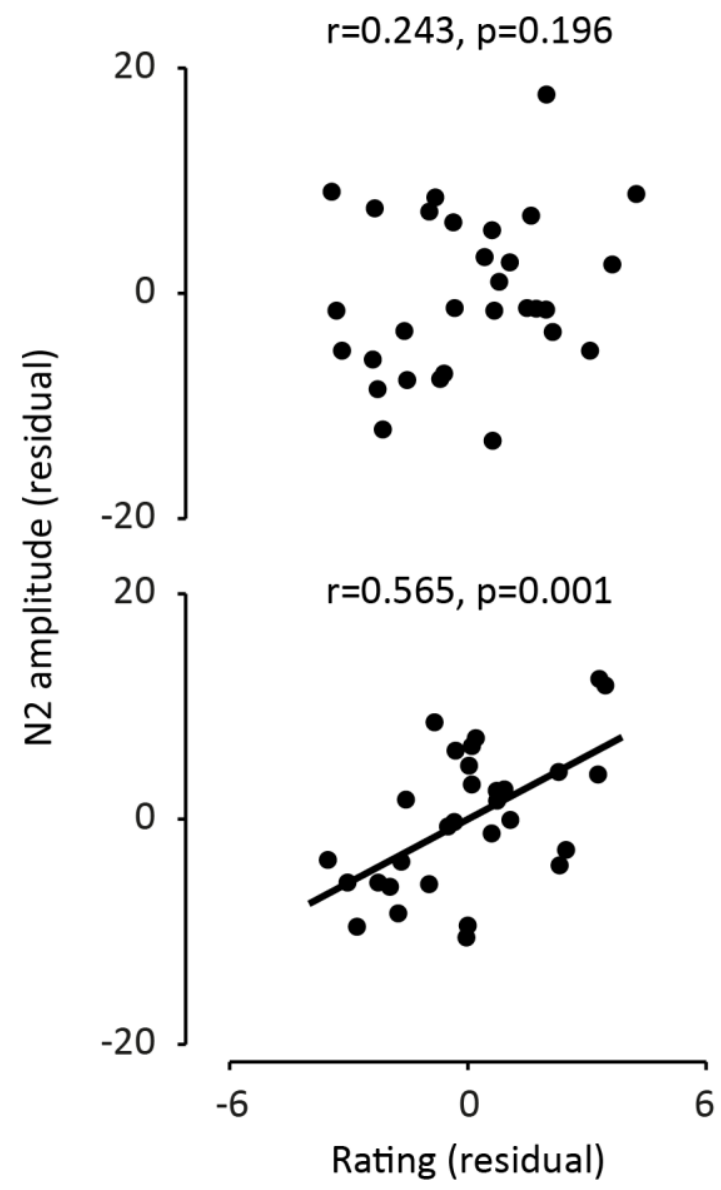

Variables controlled for: age, TIV, and skull/scalp thickness
Variables controlled for:

age, TIV, skull/scalp thickness, and contrast estimates in insular cortex 